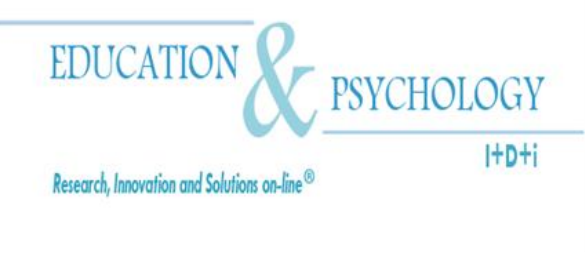

\title{
Inventario de estrategias meta-cognitivas generales (IEMG) e Inventario de estrategias meta-cognitivas en integrales (IEMI)
}

\author{
Favieri, Adriana Gladys ${ }^{1}$
}

${ }^{1}$ Departamento Aeronáutica, Universidad Tecnológica Nacional, Facultad Regional Haedo, Buenos Aires

\section{Argentina}

Correspondencia: Favieri, Adriana Gladys. Dpto de Aeronáutica. UTN FRHaedo Paris 532 (1706) Haedo, Buenos Aires, Argentina. E-mail: adriana.favieri@gmail.com

(C) Education \& Psychology I+D+i and Editorial EOS (Spain) 


\section{Resumen}

Introducción. Al hablar del conocimiento sobre el propio conocimiento, sobre cómo se percibe, se comprende, se aprende, se recuerda y se piensa, estamos hablando sobre metacognición. La misma es considerada un aspecto decisivo en el desarrollo del pensamiento reflexivo, el aprendizaje autónomo y la construcción del conocimiento. En la literatura pueden encontrarse varios instrumentos destinados a medir aspectos meta-cognitivos, sin embargo, no ha podido hallarse algún instrumento que estuviera relacionado con las estrategias metacognitivas en el área de matemática. Por lo que este estudio tiene por objetivo diseñar dos inventarios, uno sobre estrategias meta-cognitivas generales y otro sobre estrategias metacognitivas para integrales indefinidas a partir del inventario de conciencia meta-cognitiva de adultos.

Método. El estudio se centró en la traducción al castellano del inventario de conciencia metacognitiva de adultos (MAI) y su adaptación a las estrategias meta-cognitivas generales y de integrales indefinidas para elaborar dos inventarios. Se realizaron análisis factoriales utilizando el programa SPSS para analizar la validez y confiabilidad de los mismos.

Resultados. Los resultados obtenidos apoyan la validez y confiablidad de los inventarios elaborados a partir de las adaptaciones del inventario de conciencia meta-cognitiva de adultos (MAI).

Discusión y Conclusión. Los inventarios elaborados, adaptados del MAI, llamados IEMG y IEMI, son válidos y confiables para medir estrategias meta-cognitivas generales y estrategias meta-cognitivas específicas de integrales indefinidas, respectivamente.

Palabras Clave: estrategias meta-cognitivas, inventario, validez, confiabilidad, integrales indefinidas.

Recepción: 05/06/13 Aceptación inicial: 15/08/2013 Aceptación final: 08/11/13 


\title{
General Metacognitive Strategies Inventory (GMSI) and the Metacognitive Integrals Strategies Inventory (MISI)
}

\begin{abstract}
Introduction. When talking about knowledge itself, the way it is perceived, understood, learnt, remembered and thought, we are talking about metacognition. It is considered a crucial aspect in the development of reflective thought, autonomous learning and construction of knowledge. There exists several tools in literature to measure metacognitive aspects, however, there is no instrument related to metacognitive strategies in the area of mathematics. The objective of this study is to design two inventories, one on general metacognitive strategies and the other, for indefinite integrals from the inventory of metacognitive awareness of adults.
\end{abstract}

Method. This study is focused on the translation of metacognitive awareness of adults (MAI) to Spanish and its adaptation to the general metacognitive strategies and indefinite integrals to develop two inventories. Factor analyses were conducted using SPSS to analyze the validity and reliability of them.

Results. The results support the validity and reliability of inventories made from the Metacognitive Awareness Inventory (MAI).

Discussion and Conclusion. The modified versions of the MAI, called GMSI and MISI are valid and reliable for measuring general metacognitive strategies and metacognitive strategies specific for indefinite integrals, respectively.

Keywords: metacognitive strategies, inventory, validity, reliability, indefinite integrals.

Reception: 06/05/13 Initial acceptance: 08/15/13 Final acceptance: 11/08/13 


\section{Introducción}

La meta-cognición es un conocimiento sobre el propio conocimiento, sobre cómo se percibe, se comprende, se aprende, se recuerda y se piensa. Flavell (1970s), precursor de la investigación en meta-cognición dio origen al término; refiriéndose al conocimiento que uno tiene acerca de sus propios procesos y productos cognitivos, a la monitorización, la regulación y ordenación de dichos procesos en relación con los objetos cognitivos, datos o información sobre los cuales ellos influyen, normalmente al servicio de un objetivo o meta relativamente concreta (Pérez, 2006). Carrasco (1997) se refiere al conocer el qué y el cómo, mientras que Burón (1993) la define como el conocimiento que tenemos de nuestras operaciones mentales. Delmastro y Salazar (2008) consideran que en educación, la meta-cognición puede ser definida como: "una actividad consciente de pensamiento de alto nivel, que permite indagar y reflexionar sobre la forma cómo la persona aprende y controla sus propias estrategias y procesos de aprendizaje, con el objeto de modificarlos y/o mejorarlos" (p. 45).

Schraw y Moshman (1995), Brown (1996) y Baker (1991) coinciden en considerar dos componentes de la meta-cognición: el conocimiento meta-cognitivo y la regulación metacognitiva. Schraw y Moshman (1995) definen al conocimiento de la cognición como el conocimiento sobre uno mismo y sobre las propias estrategias cognitivas, que incluye tres tipos de conocimiento meta-cognitivo: el declarativo, el procedimental y el condicional. El conocimiento declarativo meta-cognitivo se refiere a saber "sobre" las cosas. El conocimiento procedimental meta-cognitivo se refiere a saber el "cómo" hacer cosas. El conocimiento condicional meta-cognitivo se refiere al "por qué” y "cuándo" de la cognición. La regulación de la cognición se refiere a las actividades meta-cognitivas que ayudan a controlar el propio pensamiento o aprendizaje. Dentro de la regulación de la cognición se distingue el planeamiento, el manejo de la información, el monitoreo de la comprensión, el control de errores y la autoevaluación posterior. El planeamiento involucra el determinar objetivos, la selección de las estrategias apropiadas y la localización de recursos necesarios para la tarea a efectuar. El manejo de la información es la secuencia de estrategias usadas para procesar la información de manera eficiente. El monitoreo de la comprensión, la evaluación del propio aprendizaje o de la estrategia usada. El control de errores, las estrategias usadas por corregir problemas de comprensión o de desempeño. La auto-evaluación posterior, se refiere a la valoración de los productos y procesos regulatorios del propio aprendizaje.

Las estrategias meta-cognitivas planifican y supervisan la acción cognitiva y tienen una doble función; de conocimiento y de control, (Beltrán y Bueno, 1995). El uso de estrate- 
gias meta-cognitivas puede apreciarse a través de la regulación y el control de las actividades realizadas durante el aprendizaje. Esta regulación y control de la comprensión se realiza a través de varias acciones. En primer lugar es necesario determinar cuáles son los objetivos de aprendizaje que se pretenden lograr, lo que requiere una reflexión consciente al encarar problemas y tomar decisiones sobre su resolución. Luego, si se advierte algún desvío con respecto a los mismos, ser capaz de reorientar o regular la acción, lo que implica un chequeo permanente del proceso de aprendizaje, en distintos momentos del mismo y para ello es necesario planificar las acciones que se llevarán a cabo, cuánto tiempo y esfuerzo se dedicará y los recursos a utilizar. Al concluir el proceso y considerar los objetivos alcanzados, se realiza una evaluación de la propia conducta para determinar si alguna decisión ha sido inapropiada o ineficaz, a fin de corregirla en futuras situaciones y conservar aquéllas que han sido útiles y eficaces (Monereo, 2001, Sevillano, 1995). En el terreno matemático, es importante que, al resolver un problema, el individuo utilice estrategias que le permitan determinar si ha obtenido una solución correcta, y si los pasos dados en el proceso de solución son también correctos (Sevillano, 1995). Por su parte, Osses (2007) define las estrategias meta-cognitivas como las acciones destinadas a conocer las propias operaciones y procesos mentales (qué), saber utilizarlas (cómo) y poder readaptarlas y/o cambiarlas de acuerdo a la tarea a realizar.

\section{Sobre la medición de las estrategias meta-cognitivas}

La medición de las estrategias meta-cognitivas puede presentar ciertas dificultades ya que forman parte de los procesos mentales del individuo. En la literatura especializada pueden encontrarse varios diseños de instrumentos destinados a este fin. Schraw y Dennison (1994), de la Universidad de Nebraska, realizaron un inventario de 52 ítems para medir la conciencia meta-cognitiva de adultos, en el que evidencian las dos componentes principales de la metacognición. O'Neil y Abedi (1996), de la Universidad del Sur de California, elaboraron un inventario para evaluar la meta-cognición en estudiantes universitarios vinculado con habilidades lectoras que ha resultado útil tanto para evaluar como para guiar a los alumnos.

En Chile, el equipo integrado por Peronard, Crespo y Velásquez (2000) ha validado un instrumento para medir meta-comprensión lectora en estudiantes de Educación General Básica y Media de la Quinta Región. Mokhtari y Reichard (2002), de la Universidad de Texas, han diseñado y validado un inventario sobre la conciencia meta-cognitiva de Estrategias de Lectura (MARSI) para lectores adolescentes y adultos. Han determinado tres factores: estrategias de lectura globales, estrategias para resolver problemas y estrategias de lectura de apo- 
yo. Alarcón, Ureña y Cárdenas (2008), de la Universidad de Granada han logrado diseñar y validar un instrumento para medir el conocimiento declarativo de la táctica en baloncesto pues consideran que el mismo es preciso para desarrollar adecuadamente destrezas en la toma de decisiones, conocimiento que el jugador va a utilizar en las situaciones de juego.

Pereira y Ramírez (2008) han evaluado el uso de estrategias meta-cognitivas lectoras de estudiantes universitarios de Venezuela, adaptando al español del Survey of Reading Strategies (SORS) (Mokhtari, et.al., 2002), diseñado para determinar el uso de estrategias metacognitivas al leer textos escolares y académicos en inglés. Los resultados indican que las estrategias más utilizadas por los sujetos son las de resolución de problemas, seguidas por las de apoyo y las globales respectivamente. En la Universidad Complutense de Madrid (España), Jiménez, Puente, Alvarado y Arrebillaga (2008), han medido estrategias meta-cognitivas sobre la conciencia lectora, utilizando un instrumento denominado ESCOLA. Han identificado alumnos con baja conciencia lectora, y sostienen que pueden diseñarse programas de intervención específicos de estrategias meta-cognitivas para la lectura.

Por su parte Dañobeitía y Ramírez (2011) de la Universidad de Talca de Chile, han diseñado y validado una batería de habilidades meta-lingüísticas siguiendo los postulados de Gombert. El propósito era incorporar la medición de la conciencia léxica, sintáctica y semántica. El instrumento da cuenta de tres factores que se corresponden con los tres tipos de habilidades evaluadas. Qun-Guan y Meng de China junto a Roehring y Mason de Estados Unidos (2011), han estudiado las propiedades psicométricas del instrumento para medir la conciencia meta-cognitiva en lectura MARSI (Mokhtari, et.al., 2002) en poblaciones de los países correspondientes. Investigaron la capacidad de lectura a través de auto-informe y medidas estandarizadas. Jaramillo y Osses (2012) validaron un instrumento sobre meta-cognición en términos de conocimiento, experiencias meta-cognitivas y autorregulación cognitiva en estudiantes de Segundo Ciclo de Educación General Básica de escuelas municipalizadas sobre comprensión lectora.

Vallejos, Jaimes, Aguilar y Merino, de la Universidades César Vallejos, Nacional Mayor de San Marcos y Nacional de la Selva, de Perú (2012), también se han dedicado a validar un inventario de estrategias meta-cognitivas en estudiantes universitarios. Por su parte Ramírez-Dorantes, Bueno-Álvarez y Echezarreta (2013) de la Universidad Autónoma de Yucatán, han traducido y validado el MSLQ (Motivated Strategies for Learning Quetisonnarie), 
instrumento de autorreporte que mide la motivación y las estrategias cognitivas, metacognitivas de aprendizaje de estudiantes. Han llamado a este instrumento Cuestionario de Motivación y Estrategias de Aprendizaje (CMEA).

Todos estos instrumentos están pensados para medir estrategias meta-cognitivas generales, muchos de ellos para las vinculadas con la comprensión lectora y uno relacionado con una actividad deportiva. Psicólogos y educadores consideran que el conocer las estrategias meta-cognitivas es un aspecto decisivo en el desarrollo del pensamiento reflexivo, del aprendizaje autónomo y de la construcción del conocimiento (Jiménez, et. al, 2009). En particular, en el desarrollo del pensamiento matemático, Schoenfeld (1994) sostiene que la forma en la que el individuo usa la información que posee al resolver un problema incluye decisiones respecto al plan a utilizar, la selección de metas o sub-metas, el monitoreo del proceso de solución y la evaluación de lo actuado. Desafortunadamente, no ha podido hallarse algún instrumento que estuviera relacionado con las estrategias meta-cognitivas que haya sido diseñado y validado el área de matemática.

\section{Objetivo}

Este estudio tiene como objetivo traducir al castellano el inventario diseñado por Schraw y Dennison (1994), llamado Metacognitive Awareness Inventory (MAI) sobre conciencia meta-cognitiva y adaptarlo a alumnos de Análisis Matemático I de carreras de Ingeniería de la Facultad Regional Haedo de la Universidad Tecnológica Nacional (Argentina), en lo referido a estrategias meta-cognitivas generales y estrategias meta-cognitivas en integrales indefinidas.

\section{Método}

\section{Participantes}

La población está compuesta por los alumnos de la asignatura Análisis Matemático I de la Facultad Regional Haedo de la Universidad Tecnológica Nacional de la provincia de Buenos Aires (Argentina). La muestra está compuesta por 278 alumnos, obtenida a partir del seguimiento de dos grupos de alumnos, con una edad promedio de 20 , que han respondido a la encuesta en forma voluntaria, de los cuales 140 corresponden al turno mañana y 138 al turno noche. El primer grupo estaba formado por 116 alumnos y el segundo constaba de 162 alumnos. 


\section{Instrumentos y procedimiento}

\section{Inventario de Schraw y Dennison}

El inventario diseñado por Schraw y Dennison en 1994, llamado Metacognitive Awareness Inventory, utiliza la técnica del auto-informe y pretende vislumbrar conciencia de los encuestados sobre el conocimiento y la regulación de la meta-cognición. Los autores aplicaron el instrumento a doscientos alumnos, y comprobaron estadísticamente la presencia de los dos factores mencionados. La validez se logró comparando los resultados con los juicios previos, acciones de monitoreo y ensayos de actuación. Han logrado una confiabilidad de $\alpha=0.90$, estableciendo que los dos factores están correlacionados a través de $r=0.54, p<0.05$. Como los resultados fueron parcialmente concluyentes, los autores reconocieron la necesidad de realizar estudios posteriores. (Peronard Thierry, Crespo Allende y Velásquez Rivera, 2000). Mientras que la mayor parte de instrumentos de medición de estrategias metacognitivas han sido diseñados para el uso con niños y adolescentes, este instrumento fue diseñado para el uso con adultos. También ha sido usado en otros estudios de meta-cognición del adulto (Hammann y Stevens, 2001; Sperling, 2004).

\section{Adaptaciones realizadas para este estudio}

El Inventario original tiene 52 ítems y se han traducido al castellano. Con el fin de adaptar este inventario a alumnos de Análisis Matemático I de carreras de Ingeniería, en lo referido a estrategias meta-cognitivas generales y estrategias meta-cognitivas en integrales indefinidas dichos ítems fueron reorganizados. Luego se realizó un análisis de las propiedades psicométricas y como resultado del mismo se descartaron algunos ítems, tomando 33 ítems para la exploración de las estrategias meta-cognitivas generales y los 16 restantes para la evaluación de las estrategias meta-cognitivas en integrales indefinidas. Así se diseñaron dos instrumentos: el inventario de estrategias meta-cognitivas generales (IEMG) y el Inventario de estrategias meta-cognitivas en integrales (IEMI).

\section{Análisis de datos}

Se llevaron a cabo análisis factoriales exploratorios de primer y segundo orden. Lo análisis de fiabilidad se calcularon mediante alpha de Crombach. En ambos casos se utilizó el programa SPSS. 


\section{Resultados}

Propiedades psicométricas de los instrumentos

Según Salkind (1999, p. 128) la validez de un constructo "es el grado en que los resultados de una prueba se relacionan con los constructos psicológicos subyacentes”. Para verificar dicha validez se hicieron análisis factoriales. Los índices del Test de esfericidad de Bartlett $(T E B)$, que indica si el modelo factorial es adecuado para la muestra en cuestión, resultaron significativos en ambos casos, lo que nos permitió rechazar la hipótesis nula de que la matriz muestral proviene de una población en que las variables no están correlacionadas. La medida de la adecuación de la muestra KMO (Kaiser-Meyer-Olkin), índice que contrasta si las correlaciones parciales entre las variables son pequeñas, resultaron significativos para los dos inventarios, sustentan la hipótesis de que las variables pueden ser explicadas por un número menor de factores. Tras haberse diseñado algún inventario, es preciso que se lleve a cabo un análisis de confiabilidad. La confiabilidad se refiere al grado en que su aplicación repetida produce los mismos resultados. Se usó el coeficiente Alpha de Cronbach, que mide la consistencia interna de los ítems del inventario, para determinar si los mismos, en el contexto de la investigación eran confiables o no (Martín y Cabero, 2008). Se detalla a continuación la metodología seguida para los dos instrumentos

\section{El inventario de estrategias meta-cognitivas generales (IEMG)}

Se introdujeron todos los ítems en el SPSS y se realizó rotación Oblimin, obteniéndose como mejor solución la que arroja de ocho factores con $42 \%$ de varianza explicada, variable que explica las variaciones respecto de la media (Morosini, 2012). 
Tabla 1. IEMG Primer análisis factorial

\begin{tabular}{|c|c|c|c|c|c|c|c|c|}
\hline Variables & Fac1 & Fac2 & Fac3 & Fac4 & Fac5 & Fac6 & Fac7 & Fac8 \\
\hline Conocimiento Fortalezas y Debilidades Intelectuales. & 0.414 & & & & & & & \\
\hline Conocimiento de Motivación & 0.320 & & & & & & & \\
\hline Conocimiento Nivel de Ansiedad & 0.374 & & & & & & & \\
\hline Conocimiento Habilid. Organiz. Información & 0.458 & & & & & & & \\
\hline Conocimiento del Control del Aprendizaje & 0.339 & & & & & & & \\
\hline Conocimiento de distintas formas de estudio & & 0.559 & & & & & & \\
\hline Uso de distintas formas de estudio & & 0.498 & & & & & & \\
\hline Conocimiento sobre localización de recursos & & 0.378 & & & & & & \\
\hline Organización del material de estudio & & 0.388 & & & & & & \\
\hline Estudio en grupo de compañeros & & 0.343 & & & & & & \\
\hline Confianza en las propias capacidades & & & 0.503 & & & & & \\
\hline Adaptación de la forma de estudio a la situación & & & 0.518 & & & & & \\
\hline Auto-motivación & & & 0.317 & & & & & \\
\hline Control del nivel de ansiedad & & & 0.588 & & & & & \\
\hline Uso de las fortalezas para compensar & & & 0.630 & & & & & \\
\hline Análisis previo & & & & 0.543 & & & & \\
\hline Lectura generalizada & & & & 0.230 & & & & \\
\hline Determinación de objetivos & & & & 0.496 & & & & \\
\hline Organización del tiempo & & & & 0.458 & & & & \\
\hline Determinación de la velocidad de estudio & & & & & 0.640 & & & \\
\hline Atención a los conceptos importantes & & & & & 0.398 & & & \\
\hline Traducción al propio lenguaje & & & & & 0.473 & & & \\
\hline Creación de ejemplos propios & & & & & 0.136 & & & \\
\hline Relación con conocimientos previos & & & & & 0.356 & & & \\
\hline Uso de diagramas & & & & & 0.462 & & & \\
\hline Chequeo Logro Parcial de Objetivos Propuestos & & & & & & 0.347 & & \\
\hline Realización Pausas para Controlar la Comprensión & & & & & & 0.309 & & \\
\hline Revisión de las Explicaciones de Clase & & & & & & & 0.655 & \\
\hline Revisión de Libros & & & & & & & 0.589 & \\
\hline Búsqueda de Ayuda Externa & & & & & & & 0.369 & \\
\hline Autoevaluación del Logro de Objetivos & & & & & & & & 0.641 \\
\hline Autoevaluación del Aprendizaje & & & & & & & & 0.652 \\
\hline Autoevaluación del Desempeño & & & & & & & & 0.594 \\
\hline
\end{tabular}

Se procede posteriormente a un análisis factorial de segundo orden, introduciendo los datos correspondientes a los ocho factores primarios. Se obtienen dos factores de segundo orden con $52 \%$ de varianza explicada, mediante rotación Oblimin que converge en tres iteraciones.

Tabla 2. IEMG Segundo análisis factorial

\begin{tabular}{lcc}
\hline \multicolumn{1}{c}{ Variables } & Fac1 & Fac2 \\
\hline Conocimiento Declarativo Meta-cognitivo & 0.66 & \\
Conocimiento Procedimental Meta-cognitivo & 0.71 & \\
Conocimiento Condicional Meta-cognitivo & 0.40 & \\
Planeamiento & & 0.56 \\
Manejo De La Información & 0.75 \\
Control De Errores & 0.62 \\
Monitoreo De La Comprensión & 0.77 \\
Autoevaluación Posterior & 0.89 \\
\hline
\end{tabular}


El Inventario de estrategias meta-cognitivas en integrales (IEMI)

Se repite el análisis factorial, con los 16 ítems restantes de la escala y se obtiene una solución con 8 factores con $72 \%$ de varianza explicada.

Tabla 3. IEMI Primer análisis factorial

\begin{tabular}{|c|c|c|c|c|c|c|c|c|}
\hline Variables & Fac1 & Fac2 & Fac3 & Fac4 & Fac5 & Fac6 & Fac7 & Fac8 \\
\hline Conocimiento Cada Procedimiento Algebraico & & & & & & & 0.76 & \\
\hline Conocimiento Cada Método Integración & & & & & & & 0.78 & \\
\hline Conocimiento Aplicación Cada Procedimiento Algebraico & & & & & & & & 0.46 \\
\hline Conocimiento Aplicación Cada Método Integración & & & & & & & & 0.86 \\
\hline Conocimiento Cuándo Aplicar Cada Procedimiento Algebraico & & & & 0.80 & & & & \\
\hline Conocimiento Cuándo Aplicar Cada Método Integración & & & & 0.83 & & & & \\
\hline Análisis Previo Distintas Formas Resolución Integral & & 0.86 & & & & & & \\
\hline Análisis Previo Distintos Procedimientos Algebraicos & & 0.87 & & & & & & \\
\hline División Estudio Método & 0.85 & & & & & & & \\
\hline Resumen Métodos Integración Aprendidos & 0.83 & & & & & & & \\
\hline Consideración Varias Alternativas Resolución Integral & & & & & & 0.53 & & \\
\hline Análisis Utilidad Método Integración Elegido & & & & & & 0.47 & & \\
\hline Cambio Procedim Algebraico Ante Resultados No Satisfactorios & & & & & 0.83 & & & \\
\hline Cambio Método Integración Ante Resultados No Satisfactorios & & & & & $0-85$ & & & \\
\hline Autoevaluación Procedimiento Algebraico Elegido & & & $0-74$ & & & & & \\
\hline Autoevaluación Método Integración Elegido & & & 0.88 & & & & & \\
\hline
\end{tabular}

Con un análisis posterior, de segundo orden, se obtienen dos factores con $40 \%$ de varianza explicada, mediante rotación Varimax que converge en tres iteraciones.

Tabla 4. IEMI Segundo análisis factorial

\begin{tabular}{lll}
\hline \multicolumn{1}{c}{ Variables } & Fac1 & Fac2 \\
\hline Conocimiento Declarativo Meta-cognitivo de Integrales & 0.69 & \\
Conocimiento Procedimental Meta-cognitivo de Integrales & 0.70 & \\
Conocimiento Condicional Meta-cognitivo de Integrales & 0.71 & \\
Planeamiento de Integrales & & 0.36 \\
Manejo De La Información de Integrales & 0.72 \\
Control De Errores de Integrales & 0.60 \\
Monitoreo De La Comprensión de Integrales & 0.47 \\
Autoevaluación Posterior de Integrales & 0.30 \\
\hline
\end{tabular}

\section{Validez}

Los valores de $K M O$ y de la prueba de Bartlett, utilizados para identificar la validez de los inventarios, fueron identificados como significativos, lo que resultó ser apropiado para el análisis de factores. El número de los factores identificado fue de 8 para los dos instrumentos. Los valores para el primer inventario resultaron $K M O=0.72 ; x^{2}=2273.51 ; p<.001$, y para el segundo, $K M O=0.66 ; x^{2}=719.6 ; p<.001$. 
Para el IEMG el Factor I incluye los ítems 26,15,20,18,13, Factor II, 23, 32, 19,24,22, Factor III, 25,14,31,29,12, Factor IV, 9,17,11,5, Factor V, 8,30,28,27,3,2, Factor VI, 21,1, Factor VII, 7, 16, 10 y Factor VIII, 6,33,4. (Ver inventario en anexo). Para el IEMI el Factor I incluye los ítems 8,9, Factor II, 7,6, Factor III, 4,3, Factor IV, 2,16, Factor V, 15,5, Factor VI, 12,11, Factor VII, 10,1 y Factor VIII, 14,13. (Ver inventario en anexo).

Mediante el segundo análisis factorial se obtiene, para el primer inventario $K M O=0.81 ; x^{2}=475.51 ; p<0.001$ y para el segundo inventario, $K M O=0.68 ; x^{2}=170.6 ; g l 28$, $p<0.001$. Para el IEMG el nuevo Factor I contiene los factores I, II y III previos, el Factor II, los IV, V, VI, VII, y VIII. Para el IEMGI el nuevo Factor I contiene los factores VII, VIII y IV previos, el Factor II, los II, I, VI, V, y III.

La solución obtenida para el primer inventario es acorde con la teoría, agrupando en el primer factor la variable Conocimiento de la cognición meta-cognitivo y en el segundo factor Regulación de la cognición meta-cognitivo. La correspondiente para el segundo inventario también resulta en dos factores, quedando así una agrupación similar a la teoría pero aplicada a integrales indefinidas; por lo que los factores se denominaron variable Conocimiento de la cognición meta-cognitivo de integrales y Regulación de la cognición meta-cognitivo de integrales, respectivamente.

\section{Confiablidad}

Los valores del coeficiente Alpha de Cronbach, usados para determinar si los inventarios en el contexto de la investigación eran confiables o no resultaron significativos. En el análisis de confiabilidad del IEMG se obtuvo un $\alpha=0.69$ para los 15 ítems correspondientes a conocimiento meta-cognitivo y un $\alpha=0.76$ para los 18 ítems correspondientes a autoregulación. El análisis correspondiente para el IEMI resultó ser de un $\alpha=0.60$ para los ítems de conocimiento meta-cognitivo de integrales y un $\alpha=0.58$ para los ítems correspondientes a auto-regulación de integrales.

Como los valores obtenidos resultaron ser mayores a 0.50 puede considerarse un nivel aceptable de confiabilidad de los instrumentos. Los dos inventarios modificados constan de dos partes con escala de Likert de cuatro opciones. Para la primera: "No lo hago nunca", Lo hago rara vez", "Lo hago a menudo", "Lo hago siempre". Y para la segunda: "Nunca", “A veces", “Casi siempre”, "Siempre”. 


\section{Discusión y Conclusiones}

La motivación de este estudio nace de la necesidad de establecer qué estrategias metacognitivas generales y específicas de integrales indefinidas tenían los alumnos de Análisis Matemático I de la Universidad Tecnológica Nacional, Facultad Regional Haedo. Desde la incorporación del término de meta-cognición por parte de Flavell (1970s) y el instrumento de medición de la misma de Schraw y Dennison (1994), se han diseñado varios nuevos instrumentos para recabar datos sobre el tema. Muchos de ellos relacionados con la comprensión lectora y sus estrategias meta-cognitivas, O'Neil y Abedi (1996), Peronard et.al. (2000), Mokhtari et.al. (2002), Pereira et.al. (2008), Jiménez et.al. (2009), Dañobeitía et.al. (2011), Qun Guan et.al. (2011), Jaramillo et.al. (2012); otros sobre estrategias meta-cognitivas en general, Vallejos et.al. (2012), Ramírez et.al. (2013) y uno sobre estrategias meta-cognitivas en el área del deporte, Alarcón et.al. (2008). Pero ninguno de ellos relacionado específicamente sobre matemática y en particular sobe integrales indefinidas.

El desarrollo y diseño del inventario de estrategias meta-cognitivas generales (IEMG) y el Inventario de estrategias meta-cognitivas en integrales (IEMI) tuvo como guía la importancia de las estrategias meta-cognitivas en el área de matemática y resolución de problemas Schoenfeld (1994) y las dos componentes principales de la meta-cognición: el conocimiento meta-cognitivo y la regulación meta-cognitiva (Schraw y Moshman, 1995, Brown, 1996 y Baker, 1991), con sus dimensiones. Los resultados obtenidos de los análisis de validación sostienen la existencia de ambas dimensiones de la meta-cognición tanto para las estrategias generales como para las específicas de integrales indefinidas.

Tras haber traducido al castellano el inventario original de 52 ítems con el fin de adaptarlo para la medición de a estrategias meta-cognitivas generales y estrategias metacognitivas en integrales indefinidas, se procedió a un análisis de las propiedades psicométricas, descartándose algunos ítems, y tomando 33 ítems para la exploración de las estrategias meta-cognitivas generales y los 16 restantes para la evaluación de las estrategias metacognitivas en integrales indefinidas. En ambos casos se trabajaron los ítems en el SPSS para realizar un primer análisis factorial y poder comprobar la existencia de ocho factores, coincidentes con las dimensiones de las componentes de la meta-cognición, las cuales se pudieron verificar a través de un segundo un análisis factorial. Consideramos importante destacar que 
se ha llegado a establecer una dimensión de conocimiento meta-cognitivo de cognición de integrales y otra sobre la regulación de la cognición de integrales.

Para el primer caso, el del inventario de las estrategias meta-cognitivas generales se han podido establecer un total de 33 ítems, de los cuales 15 corresponden al conocimiento de la cognición meta-cognitivo y 18 para la regulación de la cognición meta-cognitivo. Para el segundo inventario se ha logrado precisar 16 ítems de los cuales 6 corresponden al conocimiento de la cognición meta-cognitivo de integrales y 10 para la regulación de la cognición meta-cognitivo de integrales. Estos dos inventarios fueron administrados a 278 alumnos para poner a prueba su validez y confiabilidad. Los valores obtenidos en ambos estudios apoyan la validez y confiabilidad de los instrumentos obtenidos, el IEMG y el IEMI, a partir del inventario MAI diseñado por Schraw y Dennison en 1994.

En conclusión, este estudio reveló que las versiones modificadas del MAI, llamadas IEMG y IEMI poseen una buena confiabilidad y validez de las mediciones. Por lo tanto, se puede utilizar tanto como una herramienta de diagnóstico como de investigación para medir las estrategias meta-cognitivas generales y las específicas de integrales indefinidas de alumnos de Análisis Matemático I. En ambos inventarios se advierte la presencia de las dos dimensiones de la meta-cognición: el conocimiento de la cognición y la regulación de la cognición. A pesar de estos resultados, se considera necesario profundizar la investigación en el futuro para validar la estructura de los instrumentos con muestras más amplias y variadas.

\section{Agradecimientos}

La autora agradece a la Trad. Pública Mabel Inés Romero y a la Lic. Lidia Giromini por su colaboración en la traducción de este artículo. 


\section{Referencias}

Alarcón, F., Ureña, N. y Cárdenas, D. (2008). Diseño y validación de un instrumento de medida para el conocimiento declarativo de la táctica en baloncesto. IV Congreso Internacional y XXV Nacional de Educación Física. Recuperado de Universidad de Córdoba: http://goo.gl/yVhcva

Baker, L. (1991). Metacognition, reading, and science education. In Santa, C., and Alvermann, D. (eds.), Science Learning: Processes and Applications. International Reading Association, Newark, Delaware.

Balcinkanli, C. (2011). Inventario de Conciencia Matecognitiva para Docentes (MAIT). Electronic Journal of Research in Educational Psychology, 9(3), 1309-1332.

Beltrán, J. y Bueno, J. (1995). Psicología de la Educación. Barcelona, España: Marcombo.

Brown, A., Armbruster, B., \& Baker, L. (1986). The role of metacognition in reading and studying. In J. Orasanu (eds.), Reading comprehension from research to practice. Hillsdale, USA: Lawrence Erlbaum Associates. Doi: 10.1207/s15326985ep2303_6

Brown, N. (1996). The development of a questionnaire assessing metacognitive patterns of students majoring in accounting in higher education. Accounting Education, 15(3), 301-323. Doi: $\underline{10.1080 / 09639280600850810}$

Burón, J. (1993). Enseñar a aprender: Introducción a la metacognición. Bilbao, España: Deusto-Mensajero.

Carrasco, J. (1997). Hacia una enseñanza eficaz. Madrid, España: Ediciones Rialp.

Dañobeitía, S. y Ramírez, R. (2011). Batería de habilidades metalingüísticas: diseño y validación. Universidad de Talca, Chile. Sistemas de Bibliotecas. Recuperado de http://goo.gl/ZWkekb http://goo.gl/wQTjou

Delmastro, A. y Salazar, L. (2008). El andamiaje instruccional como activador de procesos metacognitivos durante el aprendizaje de lenguas extranjeras. Entre Lenguas, 13, 4355.

Flavell, J. (1979). Metacognition and cognitive monitoring: A new area of cognitivedevelopmental inquiry. American Psychologist, 34, 906-911. Doi: 10.1037/0003$\underline{066 X .34 .10 .906}$

Jaramillo, S. y Osses, S. (2012). Validación de un Instrumento sobre Metacognición para Estudiantes de Segundo Ciclo de Educación General Básica. Estudios pedagógicos, 38(2), 117-131. Doi: 10.4067/S0718-07052012000200008. 
Jiménez, V., Puente, A., Alvarado, J. y Arrebillaga, L. (2008). La medición de las estrategias metacognitivas mediante la Escala de Conciencia Lectora: ESCOLA. Electronic Journal of Research in Educational Psychology, 7(2), 779-804.

Martín, Q. y Cabero, M. (2008). Tratamiento estadístico de datos con SPSS. Madrid: Thomson Editores Spain.

Mokhtari, K. \& Reichard, C. (2002). Assessing Students' Metacognitive Awareness of Reading Strategies. Journal of Educational Psychology, 94(2), 249-259. Doi: $\underline{10.1037 / 0022-0663.94 .2 .249}$

Monereo, C. (2001). Estrategias de enseñanza y aprendizaje: Formación del profesorado y aplicación en la escuela (9na ed.). Barcelona, España: Graó.

Morosini, E. (2012). El concepto de varianza. Universidad Nacional de Asunción. Facultad de Filosofía. Recuperado de http://goo.gl/qHkJlp

O'Neil, H. y Abedi, J. (1996). Reliability and validity of a state metacognitive inventory: Potencial for alternative assessment. The Journal of Educational Research 89(4), 234235. Doi: $\underline{10.1080 / 00220671.1996 .9941208}$

Osses, S. (2007). Hacia un aprendizaje autónomo en el ámbito científico. Inserción de la dimensión metacognitiva en el proceso educativo. Chile: Universidad de la Frontera.

Pereira, S. y Ramírez, J. (2008). Uso de estrategias metacognitivas de estudiantes de inglés en curso pre-universitario. Revista de Pedagogía 29(85) Recuperado de: http://goo.gl/oQEG9b

Pérez, M. (2006). Enfoques en el estudio de las concepciones sobre el aprendizaje y a enseñanza. El desarrollo de la meta-cognición. En J. S. Pozo, Nuevas formas de pensar la enseñanza y el aprendizaje. Las concepciones de profesores y alumnos. (pág. 59). Barcelona, España: Graó.

Peronard, M., Crespo, N. y Velásquez, M. (2000). Evaluación del conocimiento metacomprensivo en alumnos de educación básica. Revista Signos, 33(47), 168-180. http://dx.doi.org/10.4067/S0718-09342000000100013

Qun-Guan, C., Roehring, A., Mason, R. \& Meng, W. (2011). Psychometric Properties of Meta-cognitiva Awareness of Reading Strategy Inventory. Journal of Educational and Developmental Psychology, 1(1), 3-17. Recuperado de http://goo.gl/Z4uxzF

Ramírez-Dorantes, M., Bueno-Álvarez, J. y Echezarreta, A. (2013). Validación Psicométrica del Motivated Strategies for Learning Questionnaire en Universitarios Mexicanos. Electronic Journal of Research in Educational Psychology, 11(1), 193-214.

Salkind, N. (1999). Métodos de investigación. México, México: Prentice Hall. 
Schoenfeld, A. (1994). Ideas y Tendencias en la resolución de problemas. (Separata del libro La enseñanza de la matemática a debate, publicado por el Ministerio de Educación y Ciencia. Madrid, 1985. ed.). Buenos Aires: Olimpiada Matemática Argentina.

Schraw, G., \& Dennison, R. S. (1994). Assessing metacognitive awareness. Contemporary Educational Psychology, 19(4), 460-475. Doi: 10.1006/ceps.1994.1033

Schraw, G. \& Moshman, D. (1995). Metacognitive theories. Educational Psychology Review,7(4), 351-371. http://dx.doi.org/10.1007/BF02212307

Sevillano, M. (1995). Estrategias de enseñanza y aprendizaje con medios y tecnología. Madrid, España: Centro de estudios Ramón Areces, S.A.

Vallejos, J., Jaimes, C., Aguilar, E. y Merino, M. (2012). Validez, confiabilidad y baremación del inventario de estrategias metacognitivas en estudiantes universitarios. Revista de Psicología, 14(1), 9-20. Recuperado de http://goo.gl/ZTG7dB 


\section{Anexos \\ 1. Inventario de estrategias meta-cognitivas generales (IEMG)}

Parte 1

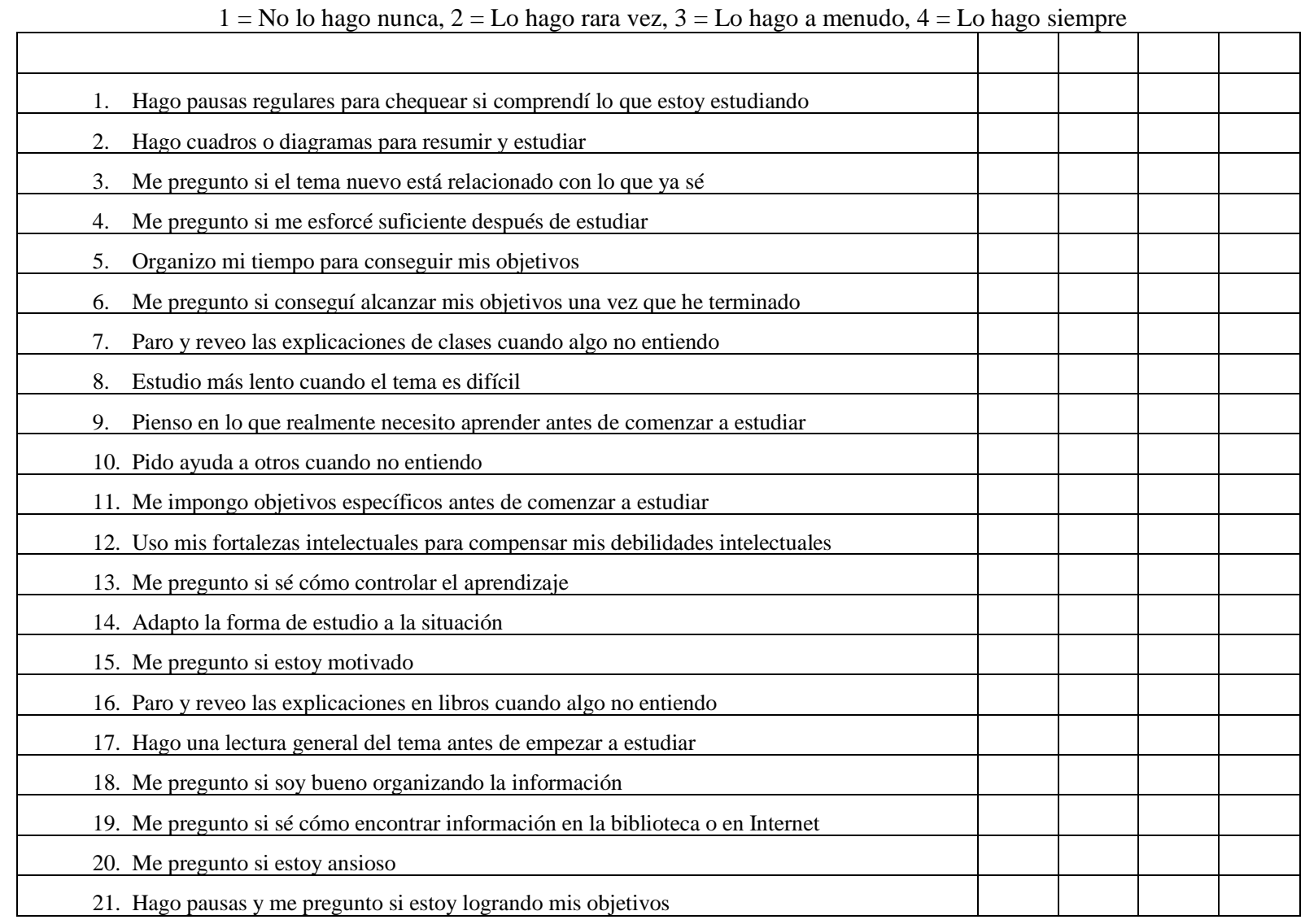

Parte 2

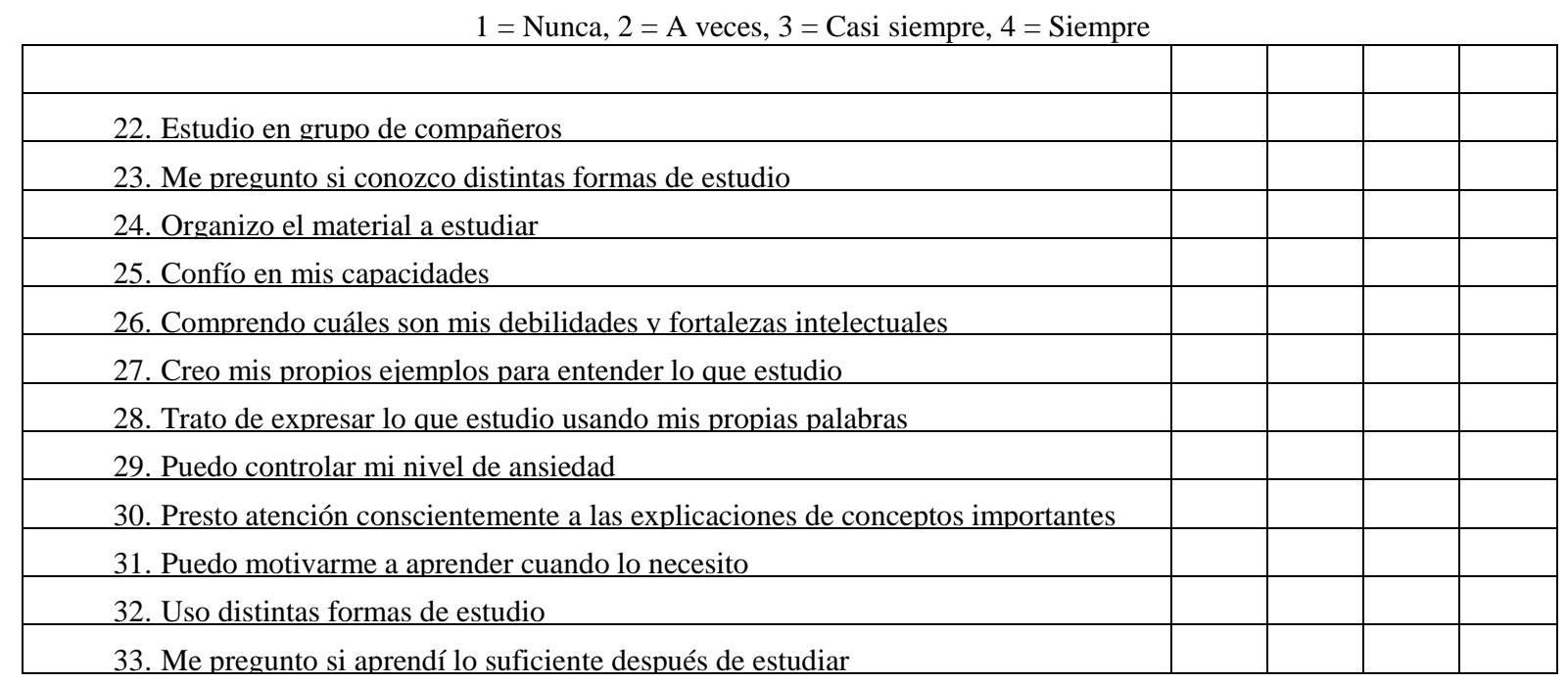




\section{Relación entre los ítems del inventario con los indicadores y las dimensiones de} las variables.

\begin{tabular}{|c|c|c|}
\hline Dimensiones & Indicadores & Ítem en IEMG \\
\hline \multirow{5}{*}{$\begin{array}{l}\text { CONOCIMIENTO DE- } \\
\text { CLARATIVO META- } \\
\text { COGNITIVO }\end{array}$} & $\begin{array}{l}\text { Conocimiento de las Propias Fortalezas y Debilida- } \\
\text { des Intelectuales }\end{array}$ & $\begin{array}{l}26 \text { Comprendo cuáles son mis debilidades y fortale- } \\
\text { zas intelectuales }\end{array}$ \\
\hline & Conocimiento de la Propia Motivación & 15 Me pregunto si estoy motivado \\
\hline & Conocimiento del Propio Nivel de Ansiedad & 20 Me pregunto si estoy ansioso \\
\hline & $\begin{array}{l}\text { Conocimiento de las Propias Habilidades Organiza- } \\
\text { tivas de la Información }\end{array}$ & $\begin{array}{l}18 \text { Me pregunto si soy bueno organizando la infor- } \\
\text { mación }\end{array}$ \\
\hline & Conocimiento del Control del Aprendizaje & 13 Me pregunto si sé cómo controlar el aprendizaje \\
\hline \multirow{5}{*}{$\begin{array}{l}\text { CONOCIMIENTO PRO- } \\
\text { CEDIMIENTAL META- } \\
\text { COGNITIVO }\end{array}$} & Conocimiento de Distintas Formas de Estudio & $\begin{array}{l}23 \text { Me pregunto si conozco distintas } \\
\text { formas de estudio }\end{array}$ \\
\hline & Uso de Distintas Formas de Estudio & 32 Uso distintas formas de estudio \\
\hline & Conocimiento sobre la Localización de Recursos & $\begin{array}{l}19 \text { Me pregunto si sé cómo encontrar información } \\
\text { en la biblioteca o en Internet }\end{array}$ \\
\hline & Organización del Material de Estudio & 24 Organizo el material a estudiar \\
\hline & Estudio en Grupo con Compañeros & 22 Estudio en grupo de compañeros \\
\hline \multirow{5}{*}{$\begin{array}{l}\text { CONOCIMIENTO CON- } \\
\text { DICIONAL META- } \\
\text { COGNITIVO }\end{array}$} & Confianza en las Propias Capacidades & 25 Confío en mis capacidades \\
\hline & Adaptación de la Forma de Estudio a la Situación & $\begin{array}{l}14 \text { Adapto la forma de estudio a la } \\
\text { situación }\end{array}$ \\
\hline & Auto-motivación & 31 Puedo motivarme a aprender cuando lo necesito \\
\hline & Control del Nivel de Ansiedad & 29 Puedo controlar mi nivel de ansiedad \\
\hline & $\begin{array}{l}\text { Uso de las Fortalezas Intelectuales en Compensación } \\
\text { de las Debilidades Intelectuales }\end{array}$ & $\begin{array}{l}12 \text { Uso mis fortalezas intelectuales para compensar } \\
\text { mis debilidades intelectuales }\end{array}$ \\
\hline \multirow{4}{*}{$\begin{array}{l}\text { PLANEAMIENTO ME- } \\
\text { TA-COGNITIVO }\end{array}$} & Análisis previo & $\begin{array}{l}9 \text { Pienso en lo que realmente necesito aprender } \\
\text { antes de comenzar a estudiar }\end{array}$ \\
\hline & Lectura generalizada & $\begin{array}{l}17 \text { Hago una lectura general del tema antes de } \\
\text { empezar a estudiar }\end{array}$ \\
\hline & Determinación de objetivos & $\begin{array}{l}11 \text { Me impongo objetivos específicos antes de } \\
\text { comenzar a estudiar }\end{array}$ \\
\hline & Organización del tiempo & 5 Organizo mi tiempo para conseguir mis objetivos \\
\hline \multirow{6}{*}{$\begin{array}{l}\text { MANEJO DE LA IN- } \\
\text { FORMACIÓN META- } \\
\text { COGNITIVO }\end{array}$} & Determinación de la velocidad de estudio & 8 Estudio más lento cuando el tema es difícil \\
\hline & Atención a los conceptos importantes & $\begin{array}{l}30 \text { Presto atención conscientemente a las explica- } \\
\text { ciones de conceptos importantes }\end{array}$ \\
\hline & Traducción al propio lenguaje & $\begin{array}{l}28 \text { Trato de expresar lo que estudio usando mis } \\
\text { propias palabras }\end{array}$ \\
\hline & Creación de ejemplos propios & $\begin{array}{l}27 \text { Creo mis propios ejemplos para entender lo que } \\
\text { estudio }\end{array}$ \\
\hline & Relación con conocimientos previos & $\begin{array}{l}3 \text { Me pregunto si el tema nuevo está relacionado } \\
\text { con lo que ya sé }\end{array}$ \\
\hline & Uso de diagramas & 2 Hago cuadros o diagramas para resumir y estudiar \\
\hline \multirow{2}{*}{$\begin{array}{l}\text { MONITOREO DE LA } \\
\text { COMPRENSIÓN } \\
\text { META-COGNITIVO }\end{array}$} & Chequeo Logro Parcial de los Objetivos Propuestos & $\begin{array}{l}21 \text { Hago pausas y me pregunto si estoy logrando } \\
\text { mis objetivos }\end{array}$ \\
\hline & $\begin{array}{l}\text { Realización de Pausas para Controlar la Compren- } \\
\text { sión }\end{array}$ & $\begin{array}{l}1 \text { Hago pausas regulares para chequear si com- } \\
\text { prendí lo que estoy estudiando }\end{array}$ \\
\hline \multirow{3}{*}{$\begin{array}{c}\text { CONTROL DE } \\
\text { ERRORES } \\
\text { META-COGNITIVO }\end{array}$} & Revisión de las Explicaciones de Clase & $\begin{array}{l}7 \text { Paro y reveo las explicaciones de clases cuando } \\
\text { algo no entiendo }\end{array}$ \\
\hline & Revisión de Libros & $\begin{array}{l}\text { 16 Paro y reveo las explicaciones en libros cuando } \\
\text { algo no entiendo }\end{array}$ \\
\hline & Búsqueda de Ayuda Externa & 10 Pido ayuda a otros cuando no entiendo \\
\hline \multirow{3}{*}{$\begin{array}{l}\text { AUTOEVALUACIÓN } \\
\text { POSTERIOR } \\
\text { META-COGNITIVO }\end{array}$} & Autoevaluación del Logro de Objetivos & $\begin{array}{l}6 \text { Me pregunto si conseguí alcanzar mis objetivos } \\
\text { una vez que he terminado }\end{array}$ \\
\hline & Autoevaluación del Aprendizaje & $\begin{array}{l}33 \text { Me pregunto si aprendí lo suficiente después de } \\
\text { estudiar }\end{array}$ \\
\hline & Autoevaluación del Desempeño & $\begin{array}{l}4 \text { Me pregunto si me esforcé suficiente después de } \\
\text { estudiar }\end{array}$ \\
\hline
\end{tabular}




\section{Inventario de estrategias meta-cognitivas en integrales (IEMI)}

Parte 1:

1 = No lo hago nunca, $2=$ Lo hago rara vez, $3=$ Lo hago a menudo, $4=$ Lo hago siempre

\begin{tabular}{|l|l|l|l|}
\hline 1. $\quad$ Cambio de procedimiento algebraico si no puedo resolver la integral & & & \\
\hline 2. Pienso distintas maneras de resolver una integral antes de empezar a hacerlo & & & \\
\hline 3. Me pregunto si sé cuándo aplicar cada procedimiento algebraico & & & \\
\hline 4. Me pregunto cuándo aplicar cada método de integración & & & \\
\hline 5. Hago un resumen de los métodos de integración aprendidos & & & \\
\hline 6. Me pregunto cómo aplicar cada procedimiento algebraico & & & \\
\hline 7. Me pregunto cómo aplicar cada método de integración & & & \\
\hline 8. Me pregunto si sé cada método de integración & & & \\
\hline 9. Me pregunto si sé cada procedimiento algebraico & & & \\
\hline
\end{tabular}

Parte 2:

$1=$ Nunca, $2=$ A veces, $3=$ Casi siempre, $4=$ Siempre

10. Cambio de método de integración si no puedo resolver la integral.

11. Analizo si el método de integración usado es útil

12. Considero varias alternativas al resolver una integral

13. Puedo determinar si el procedimiento algebraico elegido fue el apropiado

14. Puedo determinar si el método de integración elegido fue el apropiado

15. Practico las integrales por método de integración

16. Pienso distintos procedimientos algebraicos antes de comenzar a resolver la integral

4. Relación entre los ítems del inventario con los indicadores y las dimensiones de las variables.

\begin{tabular}{|c|c|c|}
\hline Dimensiones & Indicadores & Ítem en IEMI \\
\hline \multirow{2}{*}{$\begin{array}{l}\text { CONOCIMIENTO DE- } \\
\text { CLARATIVO } \\
\text { META-COGNITIVO } \\
\text { INTEGRALES }\end{array}$} & Conocimiento de Cada Método de Integración & $8 \mathrm{Me}$ pregunto si sé cada método de integración \\
\hline & Conocimiento de Cada Procedimiento Algebraico & $\begin{array}{l}9 \text { Me pregunto si sé cada procedimiento alge- } \\
\text { braico }\end{array}$ \\
\hline \multirow{2}{*}{$\begin{array}{l}\text { CONOCIMIENTO PRO- } \\
\text { CEDIMIENTAL } \\
\text { META-COGNITIVO } \\
\text { INTEGRALES }\end{array}$} & $\begin{array}{l}\text { Conocimiento sobre la Aplicación de Cada Método } \\
\text { de Integración }\end{array}$ & $\begin{array}{l}7 \text { Me pregunto cómo aplicar cada método de } \\
\text { integración }\end{array}$ \\
\hline & $\begin{array}{l}\text { Conocimiento sobre la Aplicación de Cada Procedi- } \\
\text { miento Algebraico }\end{array}$ & $\begin{array}{l}6 \text { Me pregunto cómo aplicar cada procedimien- } \\
\text { to algebraico }\end{array}$ \\
\hline \multirow{2}{*}{$\begin{array}{l}\text { CONOCIMIENTO CON- } \\
\text { DICIONAL } \\
\text { META-COGNITIVO } \\
\text { INTEGRALES }\end{array}$} & $\begin{array}{l}\text { Conocimiento sobre Cuándo Aplicar Cada Método de } \\
\text { Integración }\end{array}$ & $\begin{array}{l}4 \text { Me pregunto cuándo aplicar cada método de } \\
\text { integración }\end{array}$ \\
\hline & $\begin{array}{l}\text { Conocimiento sobre Cuándo Aplicar Cada Procedi- } \\
\text { miento Algebraico }\end{array}$ & $\begin{array}{l}3 \text { Me pregunto si sé cuándo aplicar cada proce- } \\
\text { dimiento algebraico }\end{array}$ \\
\hline \multirow{2}{*}{$\begin{array}{l}\text { PLANEAMIENTO IN- } \\
\text { TEGRALES }\end{array}$} & $\begin{array}{l}\text { Análisis Previo de las Distintas Formas de Resolu- } \\
\text { ción de una Integral }\end{array}$ & $\begin{array}{l}2 \text { Pienso distintas maneras de resolver una } \\
\text { integral antes de empezar a hacerlo }\end{array}$ \\
\hline & $\begin{array}{l}\text { Análisis Previo de Distintos Procedimiento Algebrai- } \\
\text { cos }\end{array}$ & $\begin{array}{l}16 \text { Pienso distintos procedimientos algebraicos } \\
\text { antes de comenzar a resolver la integral }\end{array}$ \\
\hline \multirow{2}{*}{$\begin{array}{l}\text { MANEJO DE LA IN- } \\
\text { FORMACIÓN INTE- } \\
\text { GRALES }\end{array}$} & División Estudio por Método & $\begin{array}{l}15 \text { Practico las integrales por método de inte- } \\
\text { gración }\end{array}$ \\
\hline & Resumen de los Métodos de Integración Aprendidos & $\begin{array}{l}5 \text { Hago un resumen de los métodos de integra- } \\
\text { ción aprendidos }\end{array}$ \\
\hline \multirow{2}{*}{$\begin{array}{l}\text { MONITOREO DE LA } \\
\text { COMPRENSIÓN INTE- } \\
\text { GRALES }\end{array}$} & $\begin{array}{l}\text { Consideración de Varias Alternativas de Resolución } \\
\text { de una Integral }\end{array}$ & $\begin{array}{l}12 \text { Considero varias alternativas al resolver una } \\
\text { integral }\end{array}$ \\
\hline & $\begin{array}{l}\text { Análisis de la Utilidad del Método de Integración } \\
\text { Elegido }\end{array}$ & $\begin{array}{l}11 \text { Analizo si el método de integración usado es } \\
\text { útil }\end{array}$ \\
\hline \multirow{2}{*}{$\begin{array}{l}\text { CONTROL DE ERRO- } \\
\text { RES INTEGRALES }\end{array}$} & $\begin{array}{l}\text { Cambio de Método de Integración Ante Resultados } \\
\text { No Satisfactorios }\end{array}$ & $\begin{array}{l}10 \text { Cambio de método de integración si no } \\
\text { puedo resolver la integral. }\end{array}$ \\
\hline & $\begin{array}{l}\text { Cambio de Procedimiento Algebraico Ante Resulta- } \\
\text { dos No Satisfactorios }\end{array}$ & $\begin{array}{l}1 \text { Cambio de procedimiento algebraico si no } \\
\text { puedo resolver la integral }\end{array}$ \\
\hline \multirow{2}{*}{$\begin{array}{l}\text { AUTOEVALUACIÓN } \\
\text { POSTERIOR INTEGRA- } \\
\text { LES }\end{array}$} & Autoevaluación del Método de Integración Elegido & $\begin{array}{l}14 \text { Puedo determinar si el método de integra- } \\
\text { ción elegido fue el apropiado }\end{array}$ \\
\hline & $\begin{array}{l}\text { Autoevaluación del Procedimiento Algebraico Elegi- } \\
\text { do }\end{array}$ & $\begin{array}{l}13 \text { Puedo determinar si el procedimiento alge- } \\
\text { braico elegido fue el apropiado }\end{array}$ \\
\hline
\end{tabular}

